\title{
Effects of a health worker-led 3-month yoga intervention on blood pressure of hypertensive patients: a randomised controlled multicentre trial in the primary care setting
}

Raja Ram Dhungana ${ }^{1 *}$, Zeljko Pedisic ${ }^{1}$, Suira Joshi², Mahesh Kumar Khanal ${ }^{2}$, Om Prakash Kalauni ${ }^{2}$, Anu Shakya ${ }^{2}$, Vijay Bhurtel ${ }^{2}$, Savyata Panthi ${ }^{2}$, K. C. Ramesh Kumar ${ }^{2}$, Binod Ghimire ${ }^{3}$, Achyut Raj Pandey ${ }^{4}$, Bihungum Bista ${ }^{5}$, Shiva Ram Khatiwoda ${ }^{6}$, Craig Steven McLachlan ${ }^{7}$ Dinesh Neupane ${ }^{8,9}$ and Maximilian de Courten ${ }^{1,10}$

\begin{abstract}
Background: Hypertension control remains a major challenge globally. A recent systematic review suggested that yoga has beneficial effects on reducing blood pressure. However, the role of yoga in hypertension management in primary health care has received little attention, and no studies have evaluated the impact of a yoga program fully delivered by health care staff on hypertension. This study, therefore, assessed the effects of a health worker-led yoga intervention on blood pressure reduction among hypertensives patients in the primary care setting.

Methods: This was a multicentric, two-arm, randomised trial conducted among hypertensive patients in seven Ayurveda Health Centres in Nepal between March 2017 and June 2018. One hundred and twenty-one participants who were on or without medications were randomised to intervention $(n=61)$ and wait-list control $(n=60)$ groups using stratified block randomisation. Participants in the intervention arm received an intervention consisting of an initial five-day structured yoga training at the centres and then a further home-based practice of yoga for five days a week for the following 90 days. Both intervention and control groups also participated in a 2-h health education session. The primary outcome of this trial was systolic blood pressure at 90-day follow-up. Data were analysed on an intention-to-treat basis using linear mixed-effects regression models.
\end{abstract}

Results: We included all 121 study participants (intervention/control $=61 / 60)$ in the primary analysis ( $52.1 \%$ males; mean \pm SD age $=47.8 \pm 10.8$ years). The difference in systolic blood pressure between the intervention group and the control group was $-7.66 \mathrm{mmHg}(95 \% \mathrm{Cl}$ : $10.4,-4.93)$. For diastolic blood pressure, the difference was -3.86 $\mathrm{mmHg}(95 \% \mathrm{Cl}:-6.65,-1.06)$. No adverse events were reported by the participants.

* Correspondence: raja.dhungana@live.vu.edu.au

${ }^{1}$ Institute for Health and Sport, Victoria University, Melbourne, Australia

Full list of author information is available at the end of the article

(c) The Author(s). 2021 Open Access This article is licensed under a Creative Commons Attribution 4.0 International License, which permits use, sharing, adaptation, distribution and reproduction in any medium or format, as long as you give appropriate credit to the original author(s) and the source, provide a link to the Creative Commons licence, and indicate if changes were made. The images or other third party material in this article are included in the article's Creative Commons licence, unless indicated otherwise in a credit line to the material. If material is not included in the article's Creative Commons licence and your intended use is not permitted by statutory regulation or exceeds the permitted use, you will need to obtain permission directly from the copyright holder. To view a copy of this licence, visit http://creativecommons.org/licenses/by/4.0/ The Creative Commons Public Domain Dedication waiver (http://creativecommons.org/publicdomain/zero/1.0/) applies to the data made available in this article, unless otherwise stated in a credit line to the data. 
(Continued from previous page)

Conclusions: A yoga program for hypertensive patients consisting of a five-day training in health centres and 90 days of practice at home is effective for reducing blood pressure. Significant benefits for hypertensive patients could be expected if such programmes would become a part of the standard treatment practice.

Trial registration: This trial was prospectively registered with the Clinical Trial Registry of India [CTRI/2017/02/ 007822] on 10/02/2017.

Keywords: Hypertension, Blood pressure, Primary care, Yoga, Ayurveda, Nepal

\section{Background}

Hypertension is a major public health problem globally affecting around $22 \%$ of the world's adult population in 2015 [1]. Together with the growing burden of hypertension, the prevalence of untreated and uncontrolled hypertensive is also very high, particularly in low-and middle- income countries (LMICs). Among all hypertensives in LMICs in 2010, 29.0\% were treated, and only $7.7 \%$ had controlled blood pressure [2]. In Nepal, for example, more than half of those who are treated still have uncontrolled blood pressure [3-5].

Beyond medication, several non-pharmacological measures are available that can contribute to the effective management of hypertension. To reduce systolic blood pressure by $4-11 \mathrm{mmHg}$ in hypertensive individuals, the new American College of Cardiology/American Heart Association guidelines recommend weight loss, "hearthealthy" diet, potassium supplementation, sodium reduction, increasing physical activity and limiting alcohol intake [6]. Using culturally accepted and evidence-based non-pharmacological measures alongside the medication may further achieve optimal control of high blood pressure in low resource primary care settings. Niu et al. [7] found that combining non-drug therapies with antihypertensive medications could further improve blood pressure reduction targets.

The use of yoga for controlling high blood pressure is an increasingly popular intervention [8-10]. It has shown positive effects not only on hypertension but also on a wide range of other health conditions [9, 11-14]. In the most recent systematic review of 49 clinical trials, Wu et al. [15] suggested that yoga is a viable antihypertensive lifestyle therapy. The findings showed that practising yoga at least three times a week is associated with a reduction in systolic blood pressure (SBP) and diastolic blood pressure (DBP) by $10 \mathrm{mmHg}$ and $6 \mathrm{mmHg}$, respectively [15]. Despite a large number of clinical trials on the effects of yoga on hypertension, there are relatively few studies conducted in primary care settings $[16,17]$, and none of them has involved existing health workers in implementing yoga programs. If yoga is to be used as an adjunct or primary initial lifestyle therapy to control hypertension in clinical settings, primary care facility-based yoga training led by clinical staff could be a feasible approach.
Given that the studies conducted in a real-world setting are more likely to be translated into practice and to minimize the gap between the evidence and practice [18, 19], this study aimed to assess the effects of structured yoga practice on blood pressure reduction among hypertensive patients in primary healthcare facilities.

\section{Methods \\ Trial design}

This was a multicentric, two-arm, randomised, wait-list controlled, nonblinded trial comparing structured yoga practice (alongside health education) against health education only over three months. It was conducted among 121 hypertensive participants in seven Ayurveda Health centres (AHCs) in Nepal between March 2017 and June 2018. The study is reported using CONSORT and Intervention Description and Replication (TIDieR) guidelines.

\section{Study participants}

Study participants were first-stage hypertensive patients attending outpatient departments at the trial centres, who had high blood pressure (SBP of $\geq 140 \mathrm{mmHg}$ and $<160 \mathrm{mmHg}$ or DBP of $\geq 85 \mathrm{mmHg}$ and $<100 \mathrm{mmHg}$ ) or had been taking antihypertensive medication with SBP of $\geq 130 \mathrm{mmHg}$ and $<160 \mathrm{mmHg}$ or DBP of $\geq 85 \mathrm{mmHg}$ and $<100 \mathrm{mmHg}$ based on clinical measurements on two occasions, 1-2 weeks apart. The criteria for participant selection were age ( $\geq 18$ years and $\leq 70$ years) irrespective of gender and medication history. Persons with diabetes, those with a known case of secondary hypertension and/ or other cardiovascular diseases/conditions, pregnant women, and those who practised yoga for 30 days or more in the previous 6 months were excluded. The hypertensive patients at each AHC were screened for eligibility criteria. Once the patients agreed to enrol in the study by providing their written informed consent, their de-identified codes and study sites were shared to the statistician (recruited outside the author team) who performed randomisation. The statistician did not have access to any other data about the participants. We used the centrally generated stratified block randomisation list to allocate the participants in the intervention and wait-list control groups, with recruiting site as a stratifying variable. A total of 121 hypertensive participants were recruited and 
allocated to intervention $(n=61)$ and control $(n=60)$ groups using the above-mentioned randomisation method. As per the published intervention protocol, the target sample size for the trial was 140 participants [20]. However, we managed to recruit 121 participants during the study period (Additional file 1). This sample size was large enough to ensure statistical power of $80 \%$ in a regression analysis with 15 independent variables (for twotailed alpha $p<0.05$ of a regression coefficient), if the true intervention effect in the population was of at least small to medium size $\left(f^{2}<0.07\right)$ according to Cohen $[21]$.

\section{Study settings}

The trial was conducted in purposively selected AHCs located in Dhading, Nuwakot, Kaski, Ramechap, Surkhet, Rolpa and Rupandehi districts. Ayurveda Health Centres (AHCs) are the primary care facilities functioning at the district level in Nepal. Currently, 61 District AHCs are in operation throughout Nepal and they provide basic preventive and curative services. One of the AHC's regular health promotion programs includes yoga training to school children and senior citizens, to promote health and wellbeing. Between 2015 and 2018, some of the health workers from AHCs were trained in yoga by the Department of Ayurveda and Alternative Medicines. Our study investigators who were also experienced yoga teachers provided training to the same health workers on the intervention package and appointed them as instructors to deliver yoga intervention to the study participants. The instructors were not certified yoga instructors, but they were trained in medical sciences, Ayurveda and yoga for three to six years. The instructors were also permanent public employees, and they agreed to implement the study without altering their usual work routines at the centres. Among the seven trial centres, AHCs located in Kaski, Nuwakot, and Surkhet were able to recruit the initially planned number of participants $(n=20)$ during the study period [20], whereas AHCs from Dhading, Ramechap, Rupandehi, and Rolpa had fewer study participants (Additional file 1).

\section{Intervention}

\section{Health Centre based five-day training}

The first component of the intervention was a five-day training delivered to the intervention group participants at the trial centres. The participants were invited to attend two-hour yoga training sessions every day for five consecutive days. The instructors (i.e. health care workers from each centre), delivered the yoga training. The wait-list control group participants received the training after the completion of the study.

\section{Two hours of health education}

In addition to the five-day yoga training session, the participants in the intervention group also received a two-hour health education session. The contents of health education were adopted from the Information, Education and Communication materials endorsed by the National Health Education Information and Communication Center, Ministry of Health and Population, Nepal. The materials contained behavioural and lifestyle modification education targeted to hypertensive participants. The wait-list control group also took part in the health education session.

\section{Home-based yoga practice}

The intervention group participants were encouraged to practise yoga at home for $30 \mathrm{~min}$ per day on five days a week, for the following 90 days from the last day of the training. They were also instructed to visit their trial centres once every 30 days for health assessment and monitoring purposes. The instructors were available over the phone, if the participants needed any help in yoga postures and procedures. The participants were also provided with recorded yoga videos with exercise instructions they could follow if needed.

The yoga program consisted of postures, breathing exercise and meditation structured for $30 \mathrm{~min}$ of practice (Additional file 2). Stretching exercise, lateral arc pose and twist pose were included in the initial $9 \mathrm{~min}$ of the session. This was followed by breathing exercises for the next $9 \mathrm{~min}$. The remaining $12 \mathrm{~min}$ were allocated for meditation and relaxation activities. Evidence suggested that postures (Asana) [22-25], breathing exercise (Pranayam) [23-26], relaxation [25, 27] and meditation [22, 23, 27] are effective for reducing hypertension while practising them in combination or individually. However, studies found the combination of posture, breathing excise and relaxation/meditation has a greater effect $[15,28]$. The yoga session in the current study, therefore, used the combined approach.

The wait-list control group did not receive yoga intervention. They required to visit the trial centres once every 30 days for routine observation.

\section{Outcomes}

SBP at follow up was the primary outcome of the study. Baseline SBP was recorded just before the intervention started and follow-up SBP was measured at $90 \pm 5$ days counting from the last day of yoga training. We used an aneroid sphygmomanometer (BP AG1-20, Microlife Corp., Taiwan) to record the blood pressure at the Outpatients Department of each trial centre. We initially recorded three blood pressure readings from the participants in each five-minute interval and then averaged the last two readings to get the final measurement. 
Alongside SBP, we also measured DBP of every individual at baseline and follow-up.

\section{Data collection}

Data were collected by face-to-face interviews, anthropometric measurements and clinical examinations. Blood pressure, body height, body weight and resting heart rate were measured at baseline and follow up. Information on socio-demographic characteristics (age, gender, marital status, ethnicity, education, occupation and income), smoking, alcohol consumption, physical activity and the use of antihypertensive medication were collected before the intervention. We applied structured questionnaires to record socio-demographic characteristics, smoking and alcohol consumption related behaviours, and seven days history of physical activity as previously described in the protocol paper in detail [20]. We measured height and weight using portable stadiometers and digital weighing machines respectively. Radial pulse was taken in the sitting position. Participants were advised to report any change in smoking, alcohol consumption and the use of medications during the study period. The data were collected by the same researcher at baseline and follow-up. The outcome assessors were aware of intervention group allocation.

\section{Data analysis}

The collected data were compiled, edited and entered in Epidata 3.1. We used Stata 16.0 (StataCorp LLC, College Station, TX, USA) to analyse the data. The analysis was performed based on the intention-to-treat (ITT) analysis. To check the distribution of missing data, we created indicator variables for missing outcome variables and dichotomised them on the basis of missing and nonmissing. Logistic regression was performed for each indicator variable to check whether missing outcome variables could be predicted by any other study variables. A separate t-test was conducted to check whether the auxiliary variables significantly varied by the missing status of the indicator variables, but none of them was associated with the missingness of data. We used Multiple Imputation by Chained Equations (MICE) model to create 10 imputed datasets (seed of 1234). The imputation included all the variables that were in the estimation model, except income and physical activity. Separate imputation models were built for SBP and DBP. The Fraction of Missing Information (FMI) and Relative Efficiency (RE) were 11.5 and $98.9 \%$ for the model with SBP and 6.5 and $99.4 \%$ for the model with DBP (Additional files 3 and 4).

Baseline characteristics are presented as absolute frequencies, percentages, medians, arithmetic means and standard deviations. We used mixed-effects linear regression with follow-up SBP and DBP as the outcome variable to analyse the intervention effect. We conducted three analyses (Models 1-3) with a dichotomous independent variable representing belonging to the intervention group ("1") or control group ("0"). The unstandardized regression coefficient (B) for this dichotomous variable represents the estimated effect of the intervention. In all three models, the trial centre was considered as a second-level variable, and it was allowed to have a random intercept. Other than that, Model 1 (main analysis) was adjusted for baseline outcome measurements (baseline SBP in the analysis with follow-up SBP as the outcome variable and baseline DBP in the analysis with follow-up DBP as the outcome variable). In Model 2, we additionally adjusted for age, gender, marital status, ethnicity, education, occupation, income, smoking, alcohol consumption, physical activity, baseline body mass index (BMI), baseline resting heart rate and antihypertensive medication. For adjusting BMI as a time-varying covariate, along with the variables from Model 2, we added 'BMI difference' (calculated as BMI difference $=$ baseline BMI - follow-up BMI) in Model 3.

For sensitivity analysis, we did a complete-case analysis. We simultaneously conducted a mediation analysis to test whether BMI had a significant mediation effect on the outcome. We conducted subgroup analyses based on the level of adherence to the protocol from the trial centre while delivering the intervention. No per-protocol analysis was done, as data on intervention compliance were not available at the individual level. A visual inspection was done to examine whether there was any interaction between covariates and intervention effects, and their average marginal (partial) effects were plotted in the marginal plots. All tests were two-tailed and $p<$ 0.05 was considered to indicate statistical significance.

\section{Fidelity assessment of the trial}

We conducted a post-intervention survey to retrospectively assess the fidelity of intervention. Fidelity assessment was performed in three domains: intervention delivery (i.e. whether the contents of the intervention were delivered in line with the protocol), intervention receipt (i.e. whether participants understood or learned intervention components) and intervention enactment (i.e. whether participants were able to perform homebased yoga practice as instructed) [29, 30]. For this purpose, information on the contents delivered during the five-day yoga training session was collected by a thorough review of the training documents, attendance sheets and log-books from each centre. The collected information was then used to calculate the actual score of content delivery using a checklist (Additional file 5). The checklist contains the names of 10 structured yoga items and 5 health education topics that were to be covered during the intervention. Covering each yoga item and 
health education topic was assigned one point, so the maximum total score was 15 points. If the trial centre included any additional yoga items other than the ones specified in the protocol they were given a negative point. The level of agreement between the contents that were actually delivered and those that were supposed to be delivered was assessed using the percentage of agreement (PoA), expressed as the ratio of the actual score of the items and topics covered in the training session (numerator) and the total score of all yoga items and health education topics as per the protocol (denominator). The percentage was expected to be at least $90 \%$ for each centre [31].

Similarly, to assess whether the participants learned the proper yoga skills during the training sessions (i.e. intervention receipt) and applied the same skills while practising at home (i.e. intervention enactment), we interviewed randomly selected $20 \%$ of participants from the intervention group using a structured questionnaire. The participants were asked whether the training provided by the instructor was sufficient for them to learn yoga properly and whether they had practised yoga at home as instructed by the trainer (i.e. as per protocol). The responses were provided on a scale from 1 to 5 , with 1 indicating low and 5 indicating high sufficiency of the training. The findings of the survey guided the sensitivity analysis.

\section{Data and safety monitoring}

A clinical doctor who led the data monitoring and quality assurance team monitored the implementation of the trial, including participant recruitment and intervention delivery. The doctor provided necessary feedback to the study team. He was also responsible for reviewing data safety and quality, and he was the first person to report missing information and errors during data collection.

Regarding participants' safety, participants were instructed to report any serious adverse events during the intervention to the researchers located in each district. These researchers together with the clinicians from their centres were responsible for reviewing and responding to any reported adverse event and for reporting it to the Principal Investigator and Ethical Review Board.

\section{Results}

\section{Intervention effects}

Data on the primary outcomes were available for a total of 118 participants (Fig. 1). Three participants, two from intervention group (males) and one (female) from control group were lost to follow-up. None of the participants reported any changes in medication, tobacco use and alcohol consumption during the study period. Participants also did not report experiencing any adverse events as a result of the intervention.

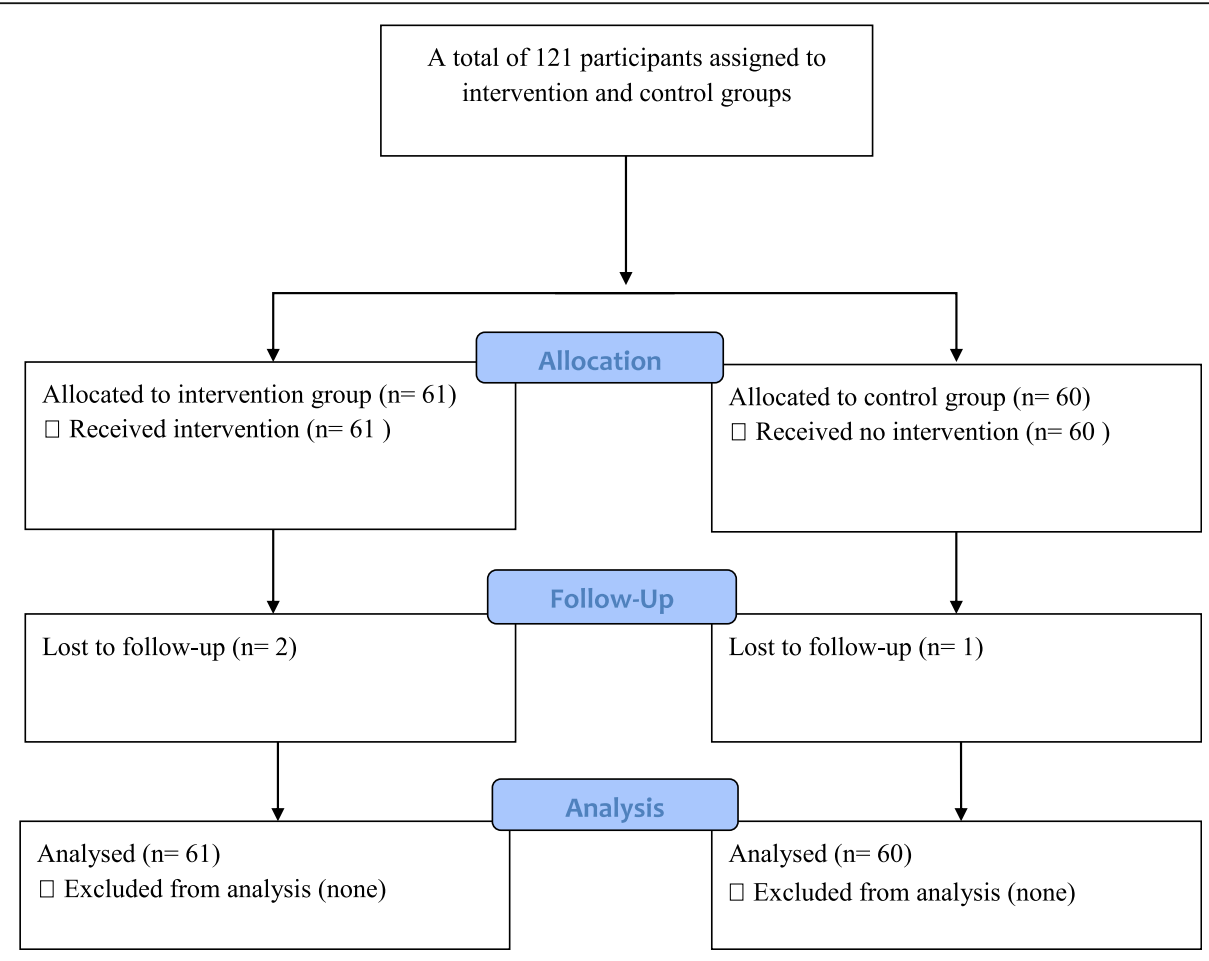

Fig. 1 Participant flow diagram 
Fifty-two percent of the participants were males. The mean (standard deviation [SD]) age of the participants was 47.7 (10.7) years. The median (interquartile range [IQR]) of years of formal education was 5 (11), where more than one third $(35.5 \%)$ of participants had no formal schooling. Smoking and alcohol consumption were reported by 14.9 and $29.7 \%$ of participants, respectively (Table 1).

The median (IQR) physical activity level expressed in MET-minutes per week was 1800 (2340) and 1530 (2580) for the intervention and control group respectively. The mean (SD) BMI was 27.4 (4.8) in $\mathrm{kg} / \mathrm{m}^{2}$. The mean (SD) of baseline SBP was $141.7(9.1) \mathrm{mmHg}$ in the intervention group and $136.9(9.0) \mathrm{mmHg}$ in the control group (Table 1). More than half $(58.7 \%)$ of the participants were on antihypertensive medications.
At follow-up, the mean (SD) of SBP was 130.1 (9.3) $\mathrm{mmHg}$ and 134.6 (11.0) $\mathrm{mmHg}$ in intervention and control group, respectively. The mean of post-intervention DBP was $84.1(6.3) \mathrm{mmHg}$ in the intervention group and 87.6 (7.0) $\mathrm{mmHg}$ in the control group (Fig. 2).

The average reduction in SBP in the intervention and control group was $11.5 \mathrm{mmHg}$ and $2.2 \mathrm{mmHg}$ respectively (Table 2). The mean reduction in DBP in the intervention and control group was $6.1 \mathrm{mmHg}$ and 1.9 $\mathrm{mmHg}$. Compared to baseline BMI and resting heart rate, the follow-up BMI and resting heart rate in the intervention group decreased by $0.37 \mathrm{~kg} / \mathrm{m}^{2}$ (vs $0.7 \mathrm{~kg} /$ $\mathrm{m}^{2}$ in control group) and 1.9 beats per minute (vs 1.2 beats per minute in control group), respectively.

In the main analysis, being in the intervention group was associated with an average $7.66 \mathrm{mmHg}(95 \% \mathrm{CI}$ :

Table 1 Baseline characteristics of the participants

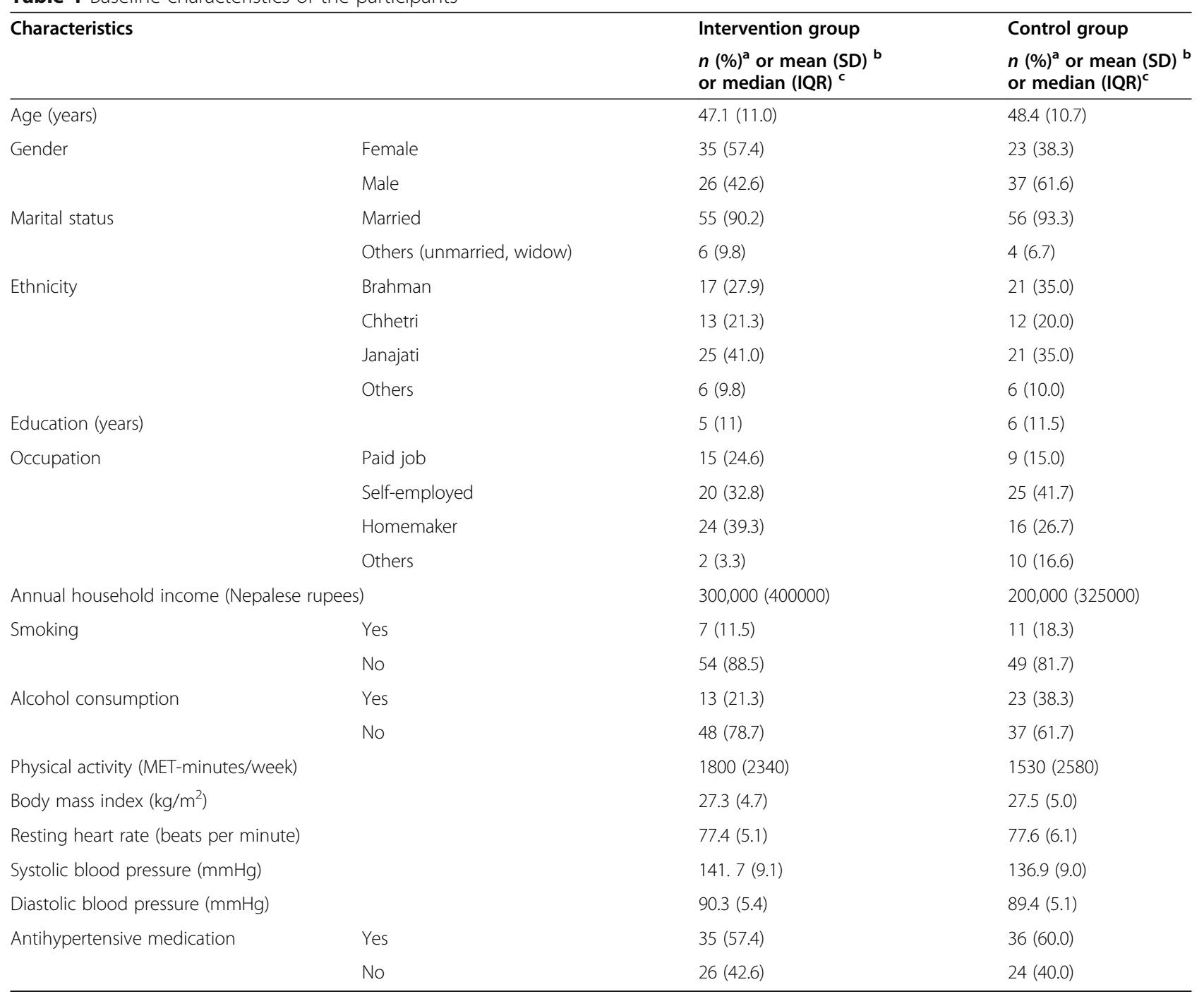

${ }^{a}$ mean and standard deviation (SD) were shown for age, body mass index, resting heart rate, systolic blood pressure and diastolic blood pressure

b number ( $n$ ) and percentage (\%) for gender, marital status, ethnicity, occupation, smoking, alcohol consumption and antihypertensive medication

c median and interquartile range (IQR) are given for education, annual household income and physical activity (moderate and vigorous activities) 

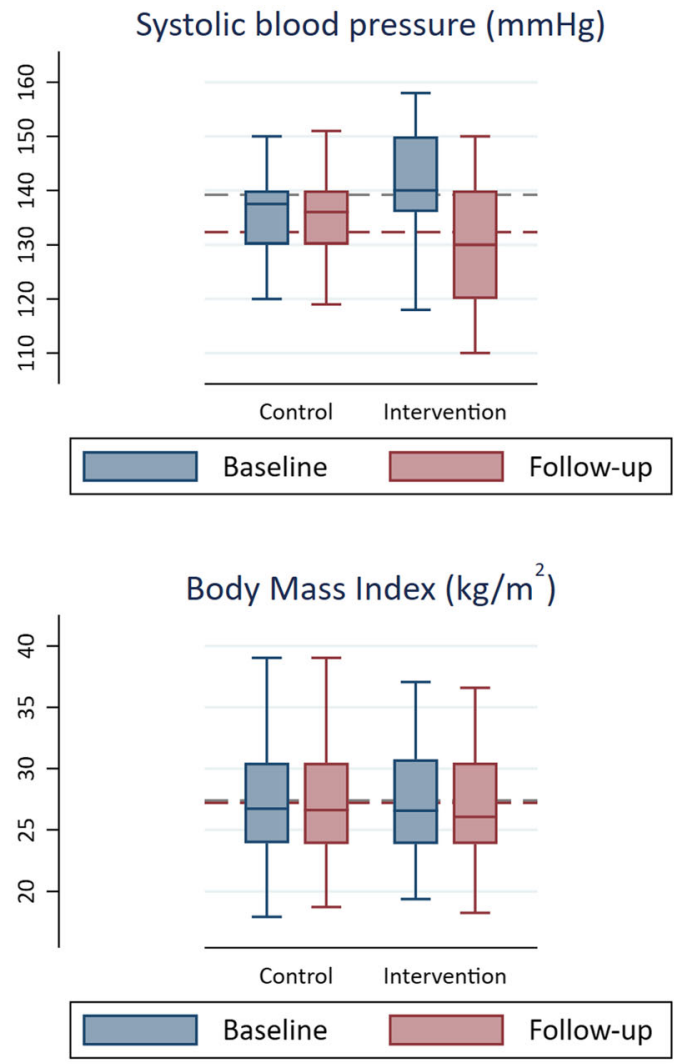

Diastolic blood pressure $(\mathrm{mmHg})$

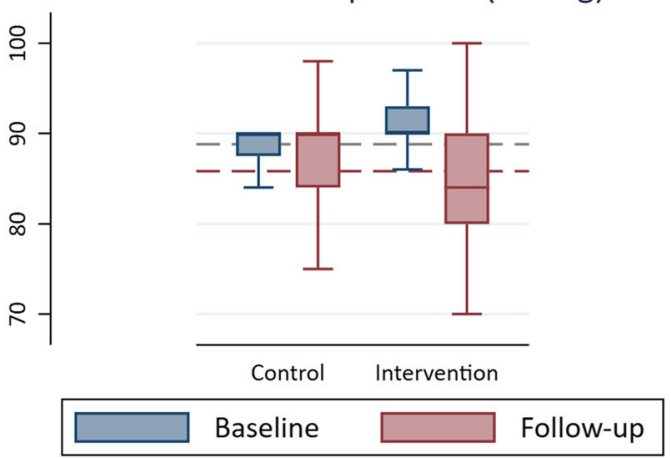

Heart rate (beats per minute)

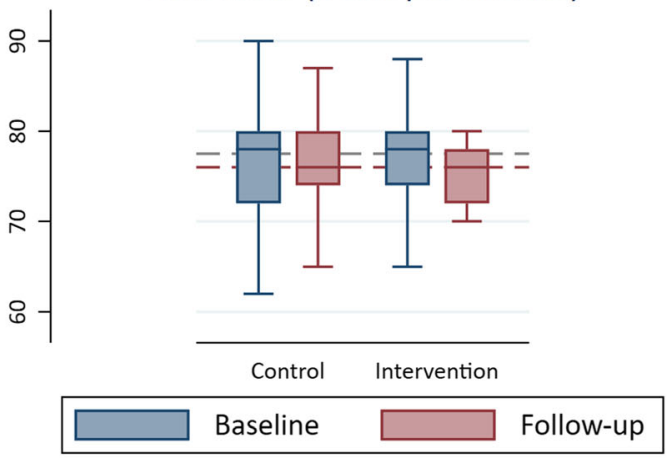

Fig. 2 Baseline and follow-up blood pressure, BMI and resting heart rate. Note: Grey dash line = Baseline mean; Maroon dash line= Follow-up mean; Box represents the inter-quartile range

4.93, 10.4) greater reduction in SBP between baseline and follow-up, compared to the control group (Table 3: Model 1). After adjusting for age, gender, ethnicity, marital status, education, occupation, household income, smoking, alcohol consumption, physical activity, baseline body mass index, antihypertensive medication, baseline resting heart rate and baseline SBP (Table 3: Model 2), being in the intervention group was associated with an average $7.41 \mathrm{mmHg}$ (95\% CI: 5.06, 9.76) greater reduction in SBP between baseline and follow-up, compared to the control group. In Model 3, the change in BMI from baseline to follow-up was significantly associated with SBP (B = - 2.49 mmHg, 95\% CI: - 3.74, - 1.20; Additional file 6$)$. The unstandardized regression coefficient (B) representing the effect of the intervention in Model 3 was -6.36 (95\% CI: $-8.63,-4.10)$, again favouring the intervention group (Table 3). Detailed outputs of Model 2 and Model 3 are provided in Additional files 6 and 7.

The reduction in DBP between baseline and follow-up was 3.86 (95\% CI: 1.06, 6.65) units higher for the intervention group, compared to the control group. In Model
2, we also found that being in the intervention group was associated with on average $3.49 \mathrm{mmHg}$ (95\% CI: $0.86,6.13)$ greater reduction in DBP between baseline and follow-up, compared with being in the control group. The unstandardized regression coefficient (B) representing the effect of the intervention in Model 3 was -2.73 (95\% CI: $-5.06,-0.41)$, again favouring the intervention group (Additional file 7).

The regression coefficients in the complete-case, sensitivity analysis (using Model 1) were nearly the same as in the main analysis ( -7.62 vs. -7.66 for SBP and -3.88 vs. -3.86 for DBP.). In mixed-effects mediation analyses, the mediating effects of the change in BMI from baseline to follow-up on the primary intervention outcomes were not significant.

The change in resting heart rate from baseline to follow-up was not significantly associated with SBD and DBP (Additional file 8). The marginal plots for gender, medication status, smoking and alcohol consumption by intervention allocation did not visually show any sign of interaction effect (Additional file 9). 
Table 2 Changes in outcome variables from baseline to follow-up

\begin{tabular}{|c|c|c|c|c|c|}
\hline \multirow[t]{2}{*}{ Characteristics } & & \multicolumn{2}{|l|}{ Change in $\mathrm{SBP}^{\mathrm{a}}$} & \multicolumn{2}{|l|}{ Change in $\mathrm{DBP}^{\mathrm{b}}$} \\
\hline & & mean & mean & mean & mean \\
\hline \multicolumn{2}{|l|}{ Total } & 11.5 & 2.3 & 6.1 & 1.8 \\
\hline \multirow[t]{2}{*}{ Gender } & Female & 10.1 & 5.0 & 5.3 & 3.9 \\
\hline & Male & 13.5 & 0.6 & 7.2 & 0.6 \\
\hline \multirow[t]{2}{*}{ Marital status } & Married & 11.5 & 1.8 & 6.0 & 1.7 \\
\hline & Others (unmarried, widow) & 10.8 & 8.3 & 7.0 & 3.8 \\
\hline \multirow[t]{4}{*}{ Ethnicity } & Brahman & 13.8 & -0.5 & 7.6 & 0.0 \\
\hline & Chhetri & 8.5 & 7.4 & 3.3 & 5.0 \\
\hline & Janajati & 11.3 & 3.2 & 6.5 & 2.4 \\
\hline & Others & 12.3 & -0.8 & 6.7 & 0.3 \\
\hline \multirow[t]{4}{*}{ Occupation } & Paid job & 11.0 & -0.4 & 5.1 & -0.6 \\
\hline & Self-employed & 13.5 & 1.0 & 7.9 & 2.4 \\
\hline & Homemakers & 9.7 & 7.1 & 5.3 & 4.7 \\
\hline & Others & 16.5 & 0.5 & 5.0 & -1.6 \\
\hline \multirow[t]{2}{*}{ Smoking } & No & 11.3 & 1.9 & 5.9 & 2.0 \\
\hline & Yes & 12.7 & 3.9 & 7.5 & 1.1 \\
\hline \multirow[t]{2}{*}{ Alcohol consumption } & No & 11.3 & 3.4 & 5.9 & 2.8 \\
\hline & Yes & 12.2 & 0.4 & 7.0 & 0.3 \\
\hline $\begin{array}{l}\text { Antihypertensive } \\
\text { Medication }\end{array}$ & No & 14.0 & 2.0 & 7.3 & 1.0 \\
\hline
\end{tabular}

${ }^{a}$ systolic blood pressure

b diastolic blood pressure

\section{Findings from fidelity assessment}

The PoA was $100 \%$ for five health centres and $90 \%$ for the two remaining centres. The difference in PoA between the health centres was not significantly associated with the primary intervention outcomes. The average score for intervention receipt was 4.3 out of five (the score provided by the participants in response to the question on whether the training was sufficient for them to learn yoga or not; Additional file 10). Similarly, 100\% of the participants reported they could perform home- based yoga practice in the same way as they were trained to do by the yoga instructor.

\section{Discussion}

In this multicentre randomised controlled trial, we found that a 3-month yoga intervention reduces systolic and diastolic blood pressure among hypertensive patients. This implies that yoga programmes can be promoted through primary care settings as an effective non-pharmacological therapy to treat hypertension.

Table 3 Intervention effects: results of multilevel mixed-effects linear regression

\begin{tabular}{|c|c|c|c|c|c|c|}
\hline \multirow[t]{2}{*}{ Outcome variable } & \multicolumn{2}{|c|}{ Model $1^{a}$} & \multicolumn{2}{|c|}{ Model $2^{b}$} & \multicolumn{2}{|c|}{ Model $3^{c}$} \\
\hline & $\mathrm{B}^{d}$ & $95 \% \mathrm{Cl}^{\mathrm{e}}$ & $B^{d}$ & $95 \% \mathrm{Cl}^{\mathrm{e}}$ & $\overline{B^{d}}$ & $95 \% \mathrm{Cl}^{\mathrm{e}}$ \\
\hline Systolic blood pressure & $-7.66^{* * *}$ & $-10.4,-4.93$ & $-7.41^{* * *}$ & $-9.76,-5.06$ & $-6.36^{* * *}$ & $-8.63,-4.10$ \\
\hline Diastolic blood pressure & $-3.86^{* *}$ & $-6.65,-1.06$ & $-3.49^{* *}$ & $-6.13,-0.86$ & $-2.73^{*}$ & $-5.06,-0.41$ \\
\hline
\end{tabular}

${ }^{a}$ Model included a dichotomous independent variable representing belonging to the intervention group (" 1 ") or control group ("0") and trial centre as a secondlevel variable, and was adjusted for baseline systolic or diastolic blood pressure (depending on the outcome variable)

b Adjusted for age, gender, marital status, ethnicity, education, occupation, income, smoking, alcohol consumption, physical activity, body mass index (BMI), resting heart rate, and baseline systolic or diastolic blood pressure (depending on the outcome variable)

c Additionally adjusted for the difference in BMI between baseline and follow-up

d Unstandardized regression coefficient

e $95 \%$ confidence interval for B

${ }^{*} p<0.05$

${ }^{* *} p<0.01$

${ }^{* * *} p<0.001$ 
Our findings are consistent with a recent systematic review that found an average reduction of SBP by $7.9 \mathrm{mmHg}$ and DBP by $4.3 \mathrm{mmHg}$ among the participants who received a yoga intervention including breathing techniques and meditation [15]. In another review, Cramer et al. found that yoga interventions lasting eight weeks or more, reduced SBP on average by $-9.65 \mathrm{mmHg}$ [32]. The pooled effect from the Cramer et al. meta-analysis may seem somewhat higher than the average effect found in our study. However, due to a relatively small pooled sample size and large heterogeneity between individual studies included in the meta-analysis, the confidence interval of the pooled effect from Cramer et al. [32] study was very wide, and it largely overlaps with our narrower confidence interval for the respective effect in our study. A smaller blood pressurelowering effect in our study compared to the Cramer et al. [32] meta-analysis might be because of the attenuation of the intervention effect due to its implementation in a realworld clinical setting. Likewise, the implementation of yoga intervention in our study was done by health workers. It might be that the effect of yoga on blood pressure reduction would be higher, if the intervention was implemented by certified yoga instructors or kinesiologists.

Studies have investigated several possible underlying mechanisms for clinical effects of yoga on hypertension [33-35]. One of the hypothesized mechanisms is that yoga affects the autonomic nervous system by stimulating activity of parasympathetic and reducing activity of sympathetic nervous system [33]. It is also postulated that yoga increases bioavailability and blood levels of nitric oxide and promotes vasodilation [33]. Additionally, participation in yoga as a "mind-body" activity has been associated with improved physiological markers, reduced symptoms of stress, and better mood [36, 37]. Pascoe et al. [36] in their systematic review concluded that mindfulness-based activities, including yoga, lead to decreased cortisol level, a stress hormone that has been linked to high blood pressure. Thoroughly investigating the mechanism of the effect of yoga on blood pressure was beyond the scope of this study. Nevertheless, we considered the possible mediating effect of the change in BMI and resting heart rate between baseline and follow-up, and we found no strong indication either of these would constitute the underlying mechanism. Given that yoga is a complex activity, it might be challenging to determine a single mechanism that would explain antihypertensive effects of all components of yoga. Therefore, to illuminate the underlying causal pathways, future studies will need to assess in detail different physiological, biomedical and stress biomarkers in relation to specific yoga components.

The main strength of the current study was that the intervention was evaluated in a real-world clinical setting. The number of such studies is generally limited. Moreover, to the best of our knowledge, this was the first study that investigated the effects of a primary health care staff-led yoga intervention on high blood pressure among the patients attending public health centres in a low-income country. One of the benefits of conducting the trial in a real-world setting is that the study could have good external validity and it could enhance the likelihood that it is translated into practice $[18,19]$. The current study has the potential to be scaled up nationwide in Nepal, as the remaining $\mathrm{AHCs}$ are also equipped with both physical and human resources to implement yoga intervention. The situation is likely to be similar in many other LMICs. In Nepal, the national policy and mechanisms of using yoga as a health promotion tool are also already in place. The Multisectoral Action Plan for Prevention and Control of NCDs (2014-2020) and Urban Health Policy (2015) integrated yoga as a strategy for NCD prevention and control. Similarly, the Department of Ayurveda and Alternative Medicines have launched yoga-based interventions such as 'Swatha Jiwan karyakram'(informal translation: Healthy Life Program) and 'Vidhaylaya yoga shiskya karyakram' (informal translation: School Yoga Education Program) in 75 districts of Nepal to promote health and wellbeing of elderly and school children. The current intervention could also be an economically viable approach, as it can utilize existing resources and can also be integrated into the ongoing program that has similar modalities, such as 'Swatha Jiwan karyakram'. However, further studies are required to test the cost-effectiveness of upscaling the program. Furthermore, the current study also had well-structured intervention packages comprising different components of yoga, including postures, breathing exercises, relaxation and meditation. Previous evidence showed that these components in combination were likely to have a better positive impact on health than individual components [15, 28]. Likewise, the session timing (i.e. $30 \mathrm{~min}$ ) and frequency (i.e. five sessions per week) were selected to be in line with the World Health Organization physical activity guidelines (i.e. 150 or more minutes a week of moderate-to-vigorous physical activity). This study had a shorter session timing compared to previous studies in which the average session time was $59.2 \mathrm{~min}$ [15]. This might have positively affected participant compliance. Lastly, as this trial was conducted in several centres, representing large geographical areas of Nepal, the findings could be generalized beyond the trial participants and centres.

The current study has some limitations. Firstly, hypertension was diagnosed based on blood pressure measured on two occasions only that were 1-2 weeks apart. Although most participants were previously diagnosed hypertensive patients, it might be that we misclassified some of the newly diagnosed participants. We did not manage to collect information on $r$ adherence to the study protocol from all participants. Evidence shows that the effect of yoga may vary depending on the frequency 
and duration of yoga practice [15]. Future studies on the effects of yoga on blood pressure should aim to collect such data, to enable conducting per-protocol analysis. Furthermore, the post-intervention blood pressure measurements were not done on the same day for all participants, as this was not feasible. It was measured between the 85th and the 95th day of the intervention, as not all participants were available for the follow-up measurement on the 90th day. Besides, the variation in the level of yoga competency of the health workers who provided training to the participants might have also influenced the study outcomes. Likewise, the pre and post-intervention data were collected by the same persons and they used aneroid blood pressure machines to assess blood pressure. That might have introduced rater bias. We did not assess long-term effects of the intervention. It might be that the intervention would not be as efficient and sustainable over a longer period, as participant compliance to the protocol would likely reduce over time. Lastly, as the study included only first-stage hypertensive patients, study findings cannot be generalised to patients with higher stages of hypertension.

\section{Conclusion}

A simple, 3-month yoga intervention delivered by health workers in primary care centres and coupled with home-based practice is effective in lowering high blood pressure among hypertensive patients. Given that the study was conducted in real-world clinical settings, our findings suggest the intervention strategy should be considered as adjuvant or initial lifestyle therapy for hypertension in primary care.

\section{Abbreviations}

AHC: Ayurveda Health Centre; BMI: Body mass index; DBP: Diastolic blood pressure; ERB: Ethical Review Board; ITT: Intention to treat; IQR: Interquartile range; MET: Metabolic Equivalent; NCD: Non-communicable disease; SBP: Systolic blood pressure; SD: Standard deviation

\section{Supplementary Information}

The online version contains supplementary material available at https://doi. org/10.1186/s12889-021-10528-y.

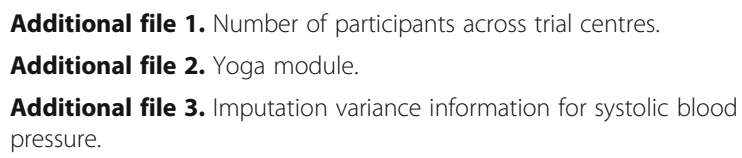

\section{Acknowledgements}

We would like to express our sincere gratitude to the district implementation team: Mr. Bishnuhari Acharya, Rupandehi; Mr. Deepak Karki, Ramechap; Dr. Sanker Rijal, Shreeram Acharya, Narendra Kathayet and Shivaraj Jaisey, Surkhet; Ms. Shova Duwadi, Dhading; Dr. Anil Yadav and Ram Krishna Paudel, Nuwakot; Laxmi Sharma and Tulsi Paudel, Kaski; Shriman Pokhrel, Gahendra Hamal, Dwarika Shah and Prem Prakash Hamal, Rolpa.

\section{Authors' contributions}

RRD conceptualised and designed the study, analysed the data, interpreted the findings, and prepared the first draft. MKH, SJ, OPK, AS, VB, SP and RKC conceptualised and implemented the study in Surkhet, Dhading, Kaski, Nuwakot, Ramechap, Rolpa and Gulmi respectively. RRD and BG developed yoga package. RRD and MKK designed the health education module. ARP, $\mathrm{BB}$ and SRK developed the implementation plan and executed the study. DN, CSM, ZP and MdC developed the data analysis plan, interpreted the findings and reviewed the draft. All authors read and approved the final manuscript.

\section{Funding}

RRD received the stipend as a Ph.D. student from Victoria University, Australia which is funded by the Australian Government Research Training Program Scholarship. The funding body has no role in the design of the study, the collection, analysis, and interpretation of data and in writing the manuscript.

\section{Availability of data and materials}

The datasets and study materials will be available from the corresponding author on request.

\section{Declarations}

\section{Ethics approval and consent to participate}

Ethical clearance was obtained from the Ethical Review Board of the Nepal Health Research Council Nepal (Ref No: 1185, 06 January 2017). Before obtaining the written consent from the participants, a team of coinvestigators approached them and informed about the purpose, objective, interventions, benefit, risk and outcome of the study. Besides, they were also informed about their rights to withdraw from the study and the privacy and confidentiality of the data. Data were stored safely and had restricted access to other than research investigators.

Consent for publication

Not applicable

\section{Competing interests}

None declared.

\section{Author details}

${ }^{1}$ Institute for Health and Sport, Victoria University, Melbourne, Australia. ${ }^{2}$ Ministry of Health, Kathmandu, Nepal. ${ }^{3}$ Nepal Ayurveda Research and Training Center, Kathmandu, Nepal. " DFID/NHSP3/MEOR, Abt Associates, Kathmandu, Nepal. ${ }^{5} \mathrm{Nepal}$ Health Research Council, Kathmandu, Nepal.

${ }^{6}$ Patanjali Ayurveda Medical College and Research Center, Dhulikhel, Nepal. ${ }^{7}$ Health Faculty, Torrens University, Sydney, Australia. ${ }^{8}$ Welch Center for Prevention, Epidemiology and Clinical Research, Department of Epidemiology, Johns Hopkins University, Baltimore, MD, USA. ${ }^{9}$ Nepal Development Society, Bharatpur, Chitwan, Nepal. ${ }^{10}$ Mitchell Institute for Education and Health Policy, Victoria University, Melbourne, Chitwan, Australia.

Received: 16 June 2020 Accepted: 28 February 2021

Published online: 20 March 2021

\section{References}

1. Global Health Observatory Data Repository [http://apps.who.int/gho/data/ view.main. NCDBPAWBv?lang=en]. Access 14 Mar 2021.

2. Mills KT, Bundy JD, Kelly TN, Reed JE, Kearney PM, Reynolds K, Chen J, He J. Global disparities of hypertension prevalence and control: a systematic analysis of population-based studies from 90 countries. Circulation. 2016; 134(6):441-50. 
3. Karmacharya BM, Koju RP, LoGerfo JP, Chan KCG, Mokdad AH, Shrestha A, Sotoodehnia N, Fitzpatrick AL. Awareness, treatment and control of hypertension in Nepal: findings from the Dhulikhel heart study. Heart Asia. 2017;9(1):1.

4. Mehata S, Shrestha N, Mehta R, Vaidya A, Rawal LB, Bhattarai N, Mishra SR. Prevalence, awareness, treatment and control of hypertension in Nepal: data from nationally representative population-based cross-sectional study. J Hypertens. 2018;36(8):1680-8.

5. Neupane D, Shrestha A, Mishra SR, Bloch J, Christensen B, McLachlan CS, Karki A, Kallestrup P. Awareness, prevalence, treatment, and control of hypertension in Western Nepal. Am J Hypertens. 2017;30(9):907-13.

6. Whelton PK, Carey RM, Aronow WS, Casey DE Jr, Collins KJ, Dennison Himmelfarb C, DePalma SM, Gidding S, Jamerson KA, Jones DW, et al. 2017 ACC/AHA/AAPA/ABC/ACPM/AGS/APhA/ASH/ASPC/NMA/PCNA guideline for the prevention, detection, evaluation, and Management of High Blood Pressure in adults: a report of the American College of Cardiology/American Heart Association task force on clinical practice guidelines. J Am Coll Cardiol. 2018;71(19):e127-248.

7. J-f N, X-f Z, Hu H-t, Wang J-j, Y-I L, Lu D-h. Should acupuncture, biofeedback, massage, qi gong, relaxation therapy, device-guided breathing, yoga and tai chi be used to reduce blood pressure?: recommendations based on high-quality systematic reviews. Complementary Therapies in Medicine. 2019;42:322-31.

8. Nahas R. Complementary and alternative medicine approaches to blood pressure reduction. Can Fam Physician. 2008;54(11):1529-33.

9. Tyagi A, Cohen M. Yoga and hypertension: a systematic review. Altern Ther Health Med. 2014;20(2):32-59.

10. Park CL, Braun T, Siegel T. Who practices yoga? A systematic review of demographic, health-related, and psychosocial factors associated with yoga practice. J Behav Med. 2015;38(3):460-71.

11. Innes KE, Bourguignon C, Taylor AG. Risk indices associated with the insulin resistance syndrome, cardiovascular disease, and possible protection with yoga: a systematic review. J Am Board Family Pract. 2005;18(6):491-519.

12. Deepa T, Sethu G, Thirrunavukkarasu N. Effect of yoga and meditation on mild to moderate essential hypertensives. J Clin Diagn Res. 2012;6(1):21-6.

13. Hartley L, Dyakova M, Holmes J, Clarke A, Lee MS, Ernst E, Rees K. Yoga for the primary prevention of cardiovascular disease. Cochrane Libr. 2014;(5): CD010072. https://doi.org/10.1002/14651858.CD010072.pub2. Accessed 14 Mar 2021.

14. McCaffrey R, Ruknui P, Hatthakit U, Kasetsomboon P. The effects of yoga on hypertensive persons in Thailand. Holist Nurs Pract. 2005;19(4):173-80.

15. Wu Y, Johnson BT, Acabchuk RL, Chen S, Lewis HK, Livingston J, Park CL, Pescatello LS. Yoga as antihypertensive lifestyle therapy: a systematic review and meta-analysis. Mayo Clin Proc. 2019.

16. Wolff M, Rogers K, Erdal B, Chalmers JP, Sundquist K, Midlov P. Impact of a short home-based yoga programme on blood pressure in patients with hypertension: a randomized controlled trial in primary care. J Hum Hypertens. 2016;30(10):599-605.

17. Wolff M, Sundquist K, Larsson Lonn S, Midlov P. Impact of yoga on blood pressure and quality of life in patients with hypertension - a controlled trial in primary care, matched for systolic blood pressure. BMC Cardiovasc Disord. 2013:13:111.

18. Glasgow RE, Lichtenstein E, Marcus AC. Why don't we see more translation of health promotion research to practice? Rethinking the efficacy-toeffectiveness transition. Am J Public Health. 2003;93(8):1261-7.

19. Curran GM, Bauer M, Mittman B, Pyne JM, Stetler C. Effectivenessimplementation hybrid designs: combining elements of clinical effectiveness and implementation research to enhance public health impact. Med Care. 2012;50(3):217-26.

20. Dhungana RR, Khanal MK, Joshi S, Kalauni OP, Shakya A, Bhrutel V, Panthi S, Kc RK, Ghimire B, Pandey AR, et al. Impact of a structured yoga program on blood pressure reduction among hypertensive patients: study protocol for a pragmatic randomized multicenter trial in primary health care settings in Nepal. BMC Complement Altern Med. 2018;18(1):207.

21. Cohen J. A power primer. Psychol Bull. 1992;112(1):155-9.

22. Murugesan R, Govindarajulu N, Bera T. Effect of selected yogic practices on the management of hypertension. Indian J Physiol Pharmacol. 2000;44(2):207-10,

23. Saptharishi L, Soudarssanane M, Thiruselvakumar D, Navasakthi D, Mathanraj S, Karthigeyan M, Sahai A. Community-based randomized controlled trial of nonpharmacological interventions in prevention and control of hypertension among young adults. Indian journal of community medicine: official publication of Indian Association of Preventive \& Social Medicine. 2009;34(4):329.
24. Subramanian $H$, Soudarssanane MB, Jayalakshmy R, Thiruselvakumar $D$, Navasakthi D, Sahai A, Saptharishi L. Non-pharmacological interventions in hypertension: a community-based cross-over randomized controlled trial. Indian J Community Med. 2011;36(3):191.

25. Mizuno J, Monteiro HL. An assessment of a sequence of yoga exercises to patients with arterial hypertension. J Bodyw Mov Ther. 2013;17(1):35-41.

26. Cohen BE, Chang AA, Grady D, Kanaya AM. Restorative yoga in adults with metabolic syndrome: a randomized, controlled pilot trial. Metab Syndr Relat Disord. 2008:6(3):223-9.

27. Patel C, North W. Randomised controlled trial of yoga and bio-feedback in management of hypertension. Lancet. 1975;306(7925):93-5.

28. Hagins M, Selfe T, Innes K. Effectiveness of yoga for hypertension: systematic review and meta-analysis. Evid Based Complement Alternat Med. 2013; 2013(649836):13. https://doi.org/10.1155/2013/649836.

29. Schulz R, Czaja SJ, McKay JR, Ory MG, Belle SH. Intervention taxonomy (ITAX): describing essential features of interventions. Am J Health Behav 2010;34(6):811-21.

30. Borrelli B. The assessment, monitoring, and enhancement of treatment Fidelity in public health clinical trials. J Public Health Dent. 2011;71(s1):S52-63.

31. Wickersham K, Colbert A, Caruthers D, Tamres L, Martino A, Erlen JA. Assessing fidelity to an intervention in a randomized controlled trial to improve medication adherence. Nurs Res. 2011;60(4):264-9.

32. Cramer H, Haller H, Lauche R, Steckhan N, Michalsen A, Dobos G. A systematic review and meta-analysis of yoga for hypertension. Am J Hypertens. 2014;27(9):1146-51.

33. McCall M. How might Yoga Work? An Overview of Potential Underlying Mechanisms. J Yoga Physical Therap. 2013:03(01). https://doi.org/10.4172/21 57-7595.1000130.

34. Riley KE, Park CL. How does yoga reduce stress? A systematic review of mechanisms of change and guide to future inquiry. Health Psychol Rev. 2015;9(3):379-96.

35. Bussing A, Michalsen A, Khalsa SB, Telles S, Sherman KJ. Effects of yoga on mental and physical health: a short summary of reviews. Evid Based Complement Alternat Med. 2012;2012:165410.

36. Pascoe MC, Thompson DR, Jenkins ZM, Ski CF. Mindfulness mediates the physiological markers of stress: systematic review and meta-analysis. J Psychiatr Res. 2017;95:156-78.

37. Pascoe MC, Bauer IE. A systematic review of randomised control trials on the effects of yoga on stress measures and mood. J Psychiatr Res. 2015;68: $270-82$.

\section{Publisher's Note}

Springer Nature remains neutral with regard to jurisdictional claims in published maps and institutional affiliations.

Ready to submit your research? Choose BMC and benefit from

- fast, convenient online submission

- thorough peer review by experienced researchers in your field

- rapid publication on acceptance

- support for research data, including large and complex data types

- gold Open Access which fosters wider collaboration and increased citations

- maximum visibility for your research: over $100 \mathrm{M}$ website views per year

At $\mathrm{BMC}$, research is always in progress.

Learn more biomedcentral.com/submissions 